\title{
Sais inorgânicos no controle em pós-colheita de Penicillium digitatum (Pers.) Sacc. em laranja
}

\author{
Anderson Eduardo Brunetto * \\ Keilor da Rosa Dorneles \\ Cassio Muller \\ Leandro José Dallagnol \\ Universidade Federal de Pelotas, Faculdade de Agronomia Eliseu Maciel \\ Trindade, CEP 96010-900, Pelotas - RS, Brasil \\ * Autor para correspondência \\ brunettoagronomo@hotmail.com
}

Submetido em 26/07/2018

Aceito para publicação em 03/11/2018

\section{Resumo}

O objetivo da pesquisa foi avaliar o efeito da aplicação dos sais inorgânicos cloreto de potássio $(\mathrm{KCl})$, hidróxido de potássio $(\mathrm{KOH})$ e silicato de potássio $\left(\mathrm{K}_{2} \mathrm{SiO}_{3}\right)$ sobre laranja no controle do bolor verde causado por Penicillium digitatum. O delineamento experimental utilizado foi inteiramente casualizado com cinco tratamentos e quatro repetições, cada uma composta por vinte frutos. Os frutos foram lavados e desinfestados, após $24 \mathrm{~h}$ foram feridos com uma agulha (2-3 $\mathrm{mm}$ de profundidade) e tratados com os sais inorgânicos ou água destilada (controle) e $2 \mathrm{~h}$ após, inoculados por meio da deposição de $15 \mu \mathrm{L}$ da suspensão de esporos de $P$. digitatum. Às 96,120 e 245 horas após a inoculação (hai) foram realizadas as avaliações de incidência do bolor verde, taxa da expansão da lesão, tamanho da lesão e da área esporulante. Às 245 hai, entre os sais, o $\mathrm{KOH}$ e o $\mathrm{K}_{2} \mathrm{SiO}_{3}$ foram os mais efetivos, afetando todas as variáveis avaliadas, reduzindo a incidência da doença em $80 \%$ e $36 \%$, respectivamente, quando comparados à testemunha. Contudo, o tratamento com o $\mathrm{KOH}$ afetou a qualidade visual dos frutos, ocasionando enrugamento e desidratação da casca. Desse modo, conclui-se que, entre os sais inorgânicos testados, o $\mathrm{K}_{2} \mathrm{SiO}_{3}\left(50 \mathrm{~g} . \mathrm{L}^{-1}\right)$ apresentou redução no bolor verde sem comprometer a qualidade visual do fruto, podendo ser incluído como uma medida complementar no manejo da doença.

Palavras-chave: Bolor verde; Citricultura; Controle de podridões; Minerais; Silício

\section{Abstract}

Inorganic salts in the postharvest control of Penicillium digitatum (Pers.) Sacc. in orange. The objective of this research was to evaluate the effect of treating oranges with potassium chloride $(\mathrm{KCl})$, potassium hydroxide $(\mathrm{KOH})$ and potassium silicate $\left(\mathrm{K}_{2} \mathrm{SiO}_{3}\right)$ for green mold caused by Penicillium digitatum. The experimental design was completely randomized with five treatments and four replications, each with twenty fruits. For the experiment, the fruits were washed and superficially disinfected. After 24 hours, the fruits were pierced with a needle (2-3 mm deep) and treated with inorganic salts or distilled water (control) and, 2 hours later, inoculated with $15 \mu \mathrm{L}$ of $P$. digitatum spore suspension. Green mold incidence, rate of 
lesion expansion, lesion size and sporulation area were evaluated at 96, 120 and 245 hours after inoculation (hai). At 245 hai, the $\mathrm{KOH}$ and $\mathrm{K}_{2} \mathrm{SiO}_{3}\left(50 \mathrm{~g} \cdot \mathrm{L}^{-1}\right)$ treatments were the most effective, affecting all evaluated variables and reducing the disease incidence by $80 \%$ and $36 \%$, respectively, compared to the control. However, the $\mathrm{KOH}$ treatment caused wrinkling and dehydrated the peel, affecting the visual quality of the fruits. In conclusion, among the tested inorganic salts, the $\mathrm{K}_{2} \mathrm{SiO}_{3}$ treatment $\left(50\right.$ g. $\left.\mathrm{L}^{-1}\right)$ reduced the green mold in the citrus without negatively effecting fruit appearance, which indicates that this treatment is an option as a complementary measure to manage the disease.

Key words: Citriculture; Control of rot; Green mold; Minerals; Silicon

\section{Introdução}

Entre as doenças em pós-colheita dos citros, o bolor verde [Penicillium digitatum (Pers.) Sacc] é a mais importante, e se encontra presente em todos os países produtores (GHOOSHKHANEH et al., 2018). $\mathrm{O}$ fungo $P$. digitatum causa podridão mole em frutos, que se inicia por pequenas anasarcas na superfície da casca que, rapidamente, progridem até tomarem todo $\mathrm{o}$ fruto. $\mathrm{O}$ fungo desenvolve um micélio branco sobre o tecido afetado, que depois é revestido por uma densa massa de esporos de cor verde. Uma vez alterada sua consistência, o fruto desprende um odor característico e torna-se impróprio para o consumo. Em adição, $P$. digitatum produz várias toxinas, como a patulina, que contamina sucos feitos de frutos parcialmente infectados pelo patógeno, provocando vários distúrbios como agitação, ulceração, vômitos e inflamação intestinal em quem os consome (FISCHER et al., 2007).

Para o controle do bolor verde em citros são recomendadas práticas culturais visando à redução do inóculo no campo, como eliminação de frutos infectados, cuidados na colheita e no transporte para evitar ferimentos e higienização constante dos equipamentos. Não obstante, o tratamento químico, com fungicidas do grupo benzimidazol e imidazol em pré e/ou pós-colheita, é o método mais utilizado (GHOOSHKHANEH et al., 2018).

Ademais, o mercado nacional e internacional de frutas tem buscado reduzir o uso de agrotóxicos em pós-colheita e estimular a adequação dos produtores e comerciantes à produção integrada de frutas (PIF) (FISCHER et al., 2007). Diante disso, a comunidade científica tem buscado formas alternativas de controle dessa doença, sendo que compostos tanto de origem biótica quanto abiótica têm demonstrado resultados promissores no controle de doenças de pós-colheita (BENATO et al., 2018). Esses compostos têm apresentado ação no patógeno de maneira direta bem como indireta pela potencialização de mecanismos de defesa do hospedeiro ao agente patogênico (SPADARO; DROBY, 2016).

Nesse contexto, o objetivo da pesquisa foi avaliar o efeito da aplicação dos sais inorgânicos cloreto de potássio, hidróxido de potássio e silicato de potássio sobre laranja no controle do bolor verde causado por P. digitatum.

Laranjas, cv. Valência, foram desinfestadas superficialmente com hipoclorito de sódio (1\%) por 120 segundos, em seguida lavadas por três vezes com água deionizada por 60 segundos e deixadas sob bancada a $25^{\circ} \mathrm{C}$ por $24 \mathrm{~h}$. Em seguida, em cada fruto foi realizado um ferimento, na região equatorial (diâmetro de 0,5 $\mathrm{mm}$ e profundidade de 2 a $3 \mathrm{~mm}$ ), com auxílio de uma agulha estéril, que foi marcado com um círculo com uma caneta esferográfica. Posteriormente, foi realizada a aplicação dos tratamentos: cloreto de potássio 15 g.L.-1 (KCL, Vetec, Brasil), hidróxido de potássio 15 g.L. $\mathrm{L}^{-1}$ $(\mathrm{KOH}$, Vetec, Brasil) e silicato de potássio nas doses 15 g.L. $\mathrm{L}^{-1}$ e 50 g.L. $\mathrm{L}^{-1}\left(\mathrm{~K}_{2} \mathrm{SiO}_{3}\right.$, PQ Corporation), através da imersão dos frutos por dois minutos nas respectivas soluções de cada composto. Como controle foram utilizados frutos tratados com água deionizada. Após o tratamento, os frutos foram deixados para secar em condições ambientais por $2 \mathrm{~h}$. Em seguida, os frutos foram colocados em bandejas plásticas $(8 \mathrm{~cm}$ de altura x $31 \mathrm{~cm}$ de largura x $50 \mathrm{~cm}$ de comprimento) e foi realizada sua inoculação por meio da deposição de $15 \mu \mathrm{L}$ da suspensão de esporos $\left(1 \times 10^{5}\right.$ conídios. $\left.\mathrm{mL}^{-1}\right) \mathrm{de}$ 
P. digitatum. O fungo foi isolado de frutos sintomáticos coletados em pomar comercial da região de Pelotas/RS e cultivado em meio de cultura batata-dextrose-agar (BDA) (Fluka, Sigma, USA) a $25 \pm 1^{\circ} \mathrm{C}$ e fotoperíodo de $12 \mathrm{~h}$. Após inoculação, os frutos foram mantidos a $24 \pm 1{ }^{\circ} \mathrm{C}$ e umidade relativa $90 \pm 5 \%$.

As variáveis avaliadas foram incidência do bolor verde, taxa da expansão da lesão $(r)$ e tamanho da lesão (TFL) e da área esporulante (TLE). A incidência da doença foi avaliada às 96, 120 e 245 horas após a inoculação (hai) e expressa em porcentagem de frutos sintomáticos em relação ao número total de frutos do tratamento. A taxa de expansão da lesão $(r)$, indicada pelo coeficiente angular da regressão linear foi calculada a partir das mensurações diárias do tamanho da lesão, até as 245 hai, utilizando paquímetro eletrônico digital (Stainless, Hardened, China). O tamanho final da lesão (TFL) foi medido às 245 hai, em todas as laranjas que apresentavam os sintomas de cada tratamento. $\mathrm{O}$ tamanho final da área esporulante (TLE) foi obtida de forma semelhante ao TFL, porém considerando somente a área com esporos do patógeno.

O delineamento experimental foi inteiramente casualizado com cinco tratamentos e quatro repetições, sendo cada repetição composta por vinte frutos. O experimento foi repetido duas vezes. Os dados foram submetidos à análise de variância (ANOVA) e as médias dos tratamentos comparadas pelo teste Tukey $(\mathrm{p} \leq 0.05)$.

Os sais inorgânicos reduziram a incidência do bolor verde, exceto o cloreto de potássio a partir das 120 hai (Figura 1). A maior redução na incidência da doença, de $100 \%$ às 96 hai até $80 \%$ às 245 hai, comparada à testemunha, foi obtida com hidróxido de potássio (Figura 1).

Contudo, o tratamento com o hidróxido de potássio afetou a qualidade visual dos frutos, ocasionando enrugamento, desidratação e branqueamento da casca. Os tratamentos com silicato de potássio, especialmente na dose de 50 g.L.-1 , reduziram a incidência da doença de $82 \%$ às 96 hai até $36 \%$ às 245 hai.

A taxa da expansão da lesão e o TFL somente foram reduzidos nos tratamentos com hidróxido de potássio, em 46 e 44\%, respectivamente, e o silicato de potássio, na dose 50 g.L $\mathrm{L}^{-1}$, em 29 e $26 \%$, respectivamente, comparados ao controle (Tabela 1). A área com esporulação do fungo somente foi reduzida, em $77 \%$, pelo tratamento com hidróxido de potássio, comparada ao controle (Tabela 1).

FIGURA 1: Incidência (\%) de bolor verde em frutos de laranja da cultivar Valencia, tratados como cloreto de potássio (KCl, 15 g.L $\left.{ }^{-1}\right)$, hidróxido de potássio $(\mathrm{KOH}, 15$ g.L.- $)$ e silicato de potássio $\left(\mathrm{K}_{2} \mathrm{SiO}_{3}, 15\right.$ g.L.- e 50 g.L.- $)$, às 96,120 e 145 h após a inoculação. Médias seguidas pela mesma letra, em cada horário de avaliação, não diferem entre si pelo teste de Tukey $(\mathrm{p} \leq 0,05)$.

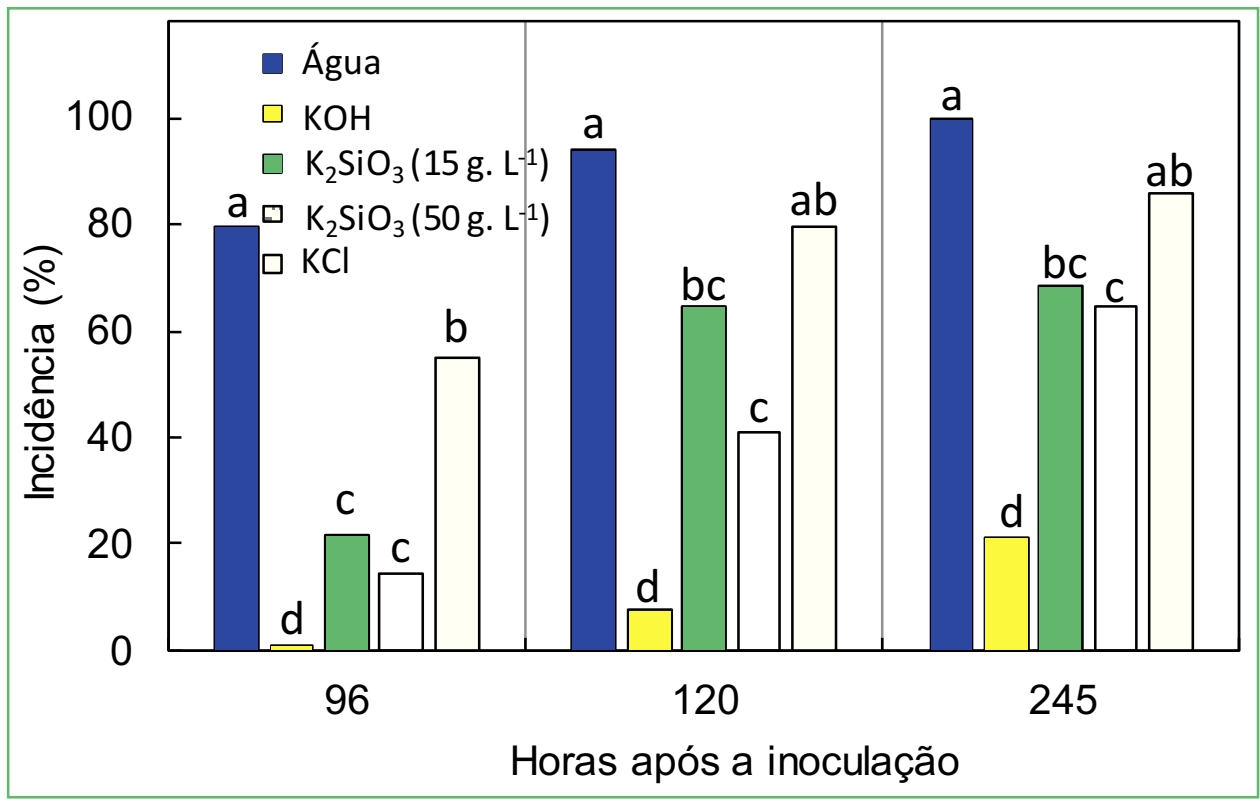


Os resultados apresentados neste estudo demonstram que o tratamento em pós-colheita com sais inorgânicos, cloreto de potássio $(\mathrm{KCl})$, hidróxido de potássio $(\mathrm{KOH})$ e silicato de potássio $\left(\mathrm{K}_{2} \mathrm{SiO}_{3}\right)$, reduziram a severidade do bolor verde causado por $P$. digitatum. Entre estes, o $\mathrm{KOH}$ seguido do $\mathrm{K}_{2} \mathrm{SiO}_{3}\left(50\right.$ g. $\left.\mathrm{L}^{-1}\right)$ foram os que apresentaram as maiores reduções na incidência e no progresso da doença.

TABELA 1: Taxa da expansão da lesão $(r)$, tamanho final da lesão (TFL) e da área esporulante (TLE) em frutos de laranja tratados com hidróxido de potássio, cloreto de potássio e silicato de potássio e inoculados com Penicillium digitatum.

\begin{tabular}{cccc}
\hline Tratamentos & $\boldsymbol{r}$ & $\begin{array}{c}\text { TFL } \\
(\mathbf{m m})\end{array}$ & $\begin{array}{c}\text { TLE } \\
(\mathbf{m m})\end{array}$ \\
\hline Controle & $0,44 \mathrm{a}$ & $46,47 \mathrm{a}$ & $42,16 \mathrm{a}$ \\
$\mathrm{KOH}$ & $0,24 \mathrm{c}$ & $26,03 \mathrm{c}$ & $9,76 \mathrm{~b}$ \\
$\mathrm{~K}_{2} \mathrm{SiO}_{3} 15$ & $0,42 \mathrm{a}$ & $46,07 \mathrm{a}$ & $35,87 \mathrm{a}$ \\
$\mathrm{K}_{2} \mathrm{SiO}_{3} 50$ & $0,31 \mathrm{~b}$ & $34,32 \mathrm{~b}$ & $29,08 \mathrm{a}$ \\
$\mathrm{KCl}$ & $0,37 \mathrm{ab}$ & $41,08 \mathrm{ab}$ & $37,80 \mathrm{a}$ \\
\hline $\mathrm{CV} \%$ & 17 & 17,25 & 34,03 \\
\hline
\end{tabular}

Tratamentos: controle (água destilada), hidróxido de potássio $\left(\mathrm{KOH}, 15 \mathrm{~g} . \mathrm{L}^{-1}\right)$, cloreto de potássio $\left(\mathrm{KCl}, 15 \mathrm{~g}\right.$. $\left.\mathrm{L}^{-1}\right)$ e silicato de potássio $\left(\mathrm{K}_{2} \mathrm{SiO}_{3}, 15 \mathrm{~g} . \mathrm{L}^{-1}\right.$ e $\left.50 \mathrm{~g} . \mathrm{L}^{-1}\right)$. Médias seguidas pela mesma letra não diferem entre si pelo teste de Tukey $(\mathrm{p} \leq 0,05)$.

O efeito adverso na infecção e colonização do patógeno causado pela aplicação dos sais é constatado através da redução da incidência e posteriormente pelo progresso de expansão da lesão causada pelo $P$. digitatum, conforme indicado pela variável $r$, que foi menor nos frutos tratados com $\mathrm{KOH} \mathrm{e} \mathrm{K}_{2} \mathrm{SiO}_{3}\left(50\right.$ g.L $\left.\mathrm{L}^{-1}\right)$. A variável $r$ se refere à velocidade de colonização do patógeno no tecido do hospedeiro, influenciando diretamente no tamanho final da lesão e na severidade final da doença (MENEGON et al., 2005). Por fim, o menor tamanho da área esporulante afeta o volume de inóculo secundário, que pode ser disperso no ambiente, dando origem a novos ciclos da doença (FISCHER et al., 2007).

Os resultados demonstram que a aplicação de sais inorgânicos em pós-colheita afetou o desenvolvimento do bolor verde. Os sais inorgânicos podem atuar contra os patógenos, por meio da alteração do $\mathrm{pH}$ e/ou aumento do potencial osmótico na superfície do hospedeiro, ambas condições prejudiciais às células fúngicas, o que podem levar ao seu colapso, comprometendo a adesão e a germinação de conídios e o desenvolvimento de hifas (DELIOPOULUS et al., 2010; JABNOUNKHIAREDDINE et al., 2016). No caso do silicato de potássio, além do provável efeito no $\mathrm{pH}$ e no potencial osmótico na superfície do hospedeiro, pode também ter a contribuição da barreira física formada pela polimerização do sal acima da cutícula, conforme já reportado em outros patossistemas (RODRIGUES; DATNOFF, 2015).

No entanto, os benefícios demonstrados pela aplicação dos sais no controle da doença podem ser mitigados devido ao comprometimento da aparência do fruto, a qual está entre os atributos de qualidade mais exigidos pelo consumidor. Dessa forma, a depreciação da qualidade visual, causada pela mudança de coloração e pelo enrugamento da casca pela aplicação do $\mathrm{KOH}$, inviabiliza o uso desse composto, na dose testada, para o controle do bolor verde em citrus.

Assim, conclui-se que, entre os sais inorgânicos testados, o silicato de potássio na dose de 50 g.L $\mathrm{L}^{-1}$ apresentou redução no bolor verde sem comprometer a qualidade visual do fruto, podendo ser incluído como uma medida complementar no manejo da doença.

\section{Referências}

BENATO, E. A.; BELLETTI, T. C.; TERAO, D.; FRANCO, D. A. $\mathrm{S}$. Óleos essenciais e tratamento térmico no controle pós-colheita de bolor verde em laranja. Summa phytopathol, Botucatu, v. 44, n. 1 , p. $65-71,2018$

DELIOPOUluS, T.; KETTLEWELL, P. S.; HARE, M. C. Fungal disease suppression by inorganic salts: a review. Crop Protection, Guildford, v. 29, n. 10, p. 1059-1075, 2010.

FISCHER, I. H.; TOFFANO, L.; LOURENÇO, S. A.; AMORIM, L. Caracterização dos danos pós-colheita em citros procedentes de "packinghouse". Fitopatologia Brasileira, Brasília, v. 32, n. 4, p. 304-310, 2007.

GHOOSHKHANEH, N. G.; GOLZARIAN, M. R.; MAMARABADI, M. Detection and classification of citrus green mold caused by Penicillium digitatum using multispectral imaging. Journal of the Science of Food and Agriculture, London, v. 98 p. 3542-3550, 2018. 
JABNOUN-KHIAREDDINE， H.; ABDALLAH, R.; ELMOHAMEDY, R.; ABDEL-KAREEM, F.; GUEDDES-CHAHED, M.; HAJLAOUI, A.; DAAMI-REMADI, M. Comparative efficacy of potassium salts against soil-borne and air-borne fungi and their ability to suppress tomato wilt and fruit rots. Journal of Microbial and Biochemical Technology, Louisville, v. 8, n. 2, p. 45-55, 2016.

MENEGON, A. P.; FORCELINI, C. A.; FERNANDES, J. M. C. Expansão de lesão por manchas foliares em cevada e sua interação com a aplicação foliar de fungicidas. Fitopatologia Brasileira, Brasília, v. 30, p. 134-138, 2005.
RODRIGUES, F. A.; DATNOFF, L. E. Silicon and plant diseases. Basel: Springer International Publishing, 2015. 148 p.

SPADARO, D.; DROBY, S. Development of biocontrol products for postharvest diseases of fruit: The importance of elucidating the mechanisms of action of yeast antagonists. Trends in Food Science \& Technology, Cambridge, v. 47, p. 39-49, 2016. 